
allemande

51-2| 2019

Les Humanités environnementales : circulations et renouvellement des savoirs en France et en Allemagne

\title{
« Notre combat pour la paix »: la France et le procès de Nuremberg (1945-1946)
}

Matthias Gemählich

\section{OpenEdition}

\section{Journals}

Édition électronique

URL : https://journals.openedition.org/allemagne/2053

DOI : $10.4000 / a l l e m a g n e .2053$

ISSN : 2605-7913

Éditeur

Société d'études allemandes

Édition imprimée

Date de publication : 10 décembre 2019

Pagination : 507-525

ISSN : 0035-0974

Référence électronique

Matthias Gemählich, « « Notre combat pour la paix » : la France et le procès de Nuremberg

(1945-1946) », Revue d'Allemagne et des pays de langue allemande [En ligne], 51-2 | 2019, mis en ligne le 02 décembre 2020, consulté le 18 mai 2021. URL : http://journals.openedition.org/allemagne/2053 DOI : https://doi.org/10.4000/allemagne.2053 


\section{«Notre combat pour la paix »: la France et le procès de Nuremberg (1945-1946)}

\section{- Matthias Gemählich *}

C'est une décision d'une portée historique que les députés de l'assemblée nationale de France prirent le 22 février 2000: ce jour-là, ils adoptèrent à une large majorité une loi permettant la ratification du statut de la Cour pénale internationale $(\mathrm{CPI})^{(1)}$. Avant le vote, le ministre des Affaires étrangères Hubert Védrine fit un discours dans lequel il souligna la volonté exprimée du gouvernement d'adhérer à la CPI et dans lequel il se déclara favorable à la ratification proposée. La création de la nouvelle juridiction internationale était, selon lui, « une vraie victoire de la lutte contre l'impunité au crépuscule d'un siècle marqué par des horreurs " ${ }^{(2)}$. Ainsi, le ministre voyait clairement la dimension historique dans laquelle le vote des députés s'inscrivait et il fit aussi référence à l'événement qui, sur le plan international, constitua la première tentative de traduire en justice les dirigeants de tout un pays et qui, depuis, symbolise le début de la lutte contre l'impunité: le procès de Nuremberg.

Mais tout en rappelant les faits historiques, Védrine n'évoqua que de manière sommaire l'œuvre des "vainqueurs de l'Allemagne nazie » ${ }^{(3)}$ et la mise en place par eux de la première juridiction internationale, le Tribunal militaire international (TMI), pour réprimer les crimes nazis, sans mentionner la participation de la France dans cette entreprise. Ce n'est ni par négligence, ni pour rester dans une perspective internationale que Védrine ne mentionna pas la contribution de son propre pays à ce célèbre procès. En fait, le discours de Védrine est révélateur de la façon dont le procès de

* Docteur en histoire contemporaine, enseignant-chercheur à l'Université Johannes Gutenberg de Mayence.

1 Assemblée nationale, $2^{\mathrm{e}}$ séance du 22 février 2000: discussion du projet de loi autorisant la ratification de la convention portant statut de la Cour pénale internationale, p. 1096. Disponible sur: http://www. assemblee-nationale.fr/11/cri/html/20000130.asp.

2 Ibid.

3 Ibid. 
Nuremberg a été perçu en France: on y a vu un événement relevant de la politique internationale, ne concernant pas ou peu le pays, et qui ne fait par conséquent pas partie de l'histoire nationale. On pourrait citer bien d'autres exemples étayant ce constat. Dans son documentaire Nuremberg - les nazis face à leurs crimes, Christian Delage laisse complètement de côté le travail de la délégation française à Nuremberg ${ }^{(4)}$. Lors des commémorations de l'ouverture du procès, la presse française rappelle régulièrement «l'héritage» du TMI et souligne que les «fondements de la justice internationale » ${ }^{(5)}$ auraient été établis à Nuremberg, mais toujours sans prendre en considération la contribution française. Dans son livre sur le procès, l'historien François Delpla affirme expressément son désintérêt pour le rôle de la France et informe le lecteur dans l'introduction que «le point de vue américain a été privilégié» ${ }^{(6)}$.

Il suffit pourtant de jeter un bref regard sur ce qui se passa entre 1945 et en 1946 à Nuremberg pour réaliser que le point de vue français est loin d'être négligeable, notamment si l'on veut comprendre le déroulement du procès devant le TMI et ses conséquences pour la codification du futur droit pénal international. Comme le tribunal de Nuremberg fut composé par les quatre puissances alliées, les représentants $\mathrm{du}$ gouvernement français eurent une influence manifeste sur l'accusation contre les dirigeants nazis et sur le verdict rendu à la fin du procès. Le présent article a pour but d'éclairer cet aspect longtemps négligé par l'historiographie et d'examiner le rôle de la France au procès de Nuremberg sous différents angles ${ }^{(7)}$. Dans ce contexte, il est tout d'abord important de montrer dans quelle mesure le Gouvernement provisoire suivit au printemps 1945 les préparatifs du procès contre les dirigeants nazis et quelles positions il adopta lors des négociations avec les autres puissances alliées. Sur cette base, il semble nécessaire d'examiner la composition et le fonctionnement de la délégation qui fut créée pour la durée du procès de Nuremberg et qui y représentait la France. Nombreuses sont aussi les questions qui concernent directement le procès: quelles furent la stratégie et l'argumentation exacte de l'accusation française devant le TMI? Quelle valeur peut-on accorder aux preuves qu'elle a produites? Y avait-il des différends avec les autres délégations alliées? Quel fut l'impact de l'accusation française sur les condamnations individuelles? En fin de compte, la perception du procès de Nuremberg en France est aussi à prendre en considération: quelle fut la réaction de l'opinion publique? Quelles ont été les conséquences politiques du verdict du TMI ? Ces questions sont examinées dans le présent article qui repose principalement sur une exploitation du fonds d'archives françaises sur le procès de Nuremberg.

4 Christian Delage, Nuremberg - Les nazis face à leurs crimes (2006).

5 «Le procès de Nuremberg 65 ans après », Le Monde, 20 novembre 2010.

6 François Delpla, Nuremberg face à l'histoire, Paris, l'Archipel, 2006, p. 8. Peu nombreux sont les ouvrages qui s'intéressent à la contribution française; voir Antonin Tisseron, La France et le procès de Nuremberg. Inventer le droit international, Paris, Prairies Ordinaires, 2014; Annette WieviorkA, Le procès de Nuremberg, Paris, Liana Levi, 2006.

7 L'article résume les résultats de la thèse de doctorat de l'auteur rédigée en allemand: Matthias Gem ̈̈нLICH, Frankreich und der Nürnberger Prozess gegen die Hauptkriegsverbrecher 1945/46, Francfort-sur-le-Main, Peter Lang, 2018. 


\section{La préparation du procès de Nuremberg}

Dans le débat sur le sort des "grands criminels de guerre» allemands, donc des représentants du régime nazi dont les crimes, d’après la déclaration de Moscou de 1943, ne pouvaient pas être géographiquement localisés, longtemps le Gouvernement provisoire de la République française n'intervint pas activement et se borna à prendre connaissance des propositions des autres gouvernements alliés. Le premier projet concret qui montre une prise de position française date de février 1945: il s'agit d'une note rédigée par Jacques Fouques-Duparc, directeur du Secrétariat des conférences au Quai d'Orsay, et signée par Georges Bidault, ministre des Affaires étrangères ${ }^{(8)}$. Dans ce document, l'idée de traduire les grands criminels de guerre en justice et de créer un tribunal international à cette fin est approuvée sans réserve.

Tout en préconisant la mise en œuvre d'une procédure judiciaire pour juger les crimes de l'Allemagne nazie sur le plan international, Fouques-Duparc avertit du «danger d'inefficacité d'une inculpation ne reposant pas sur un droit positif certain ${ }^{(9)}$. C'est pourquoi il voulait limiter l'accusation contre les dirigeants nazis, devant un tribunal international, aux atrocités et exactions allemandes commises pendant et, éventuellement, aussi avant la guerre, donc aux crimes de guerre au sens propre et à ce qu'on appellerait plus tard les «crimes contre l'humanité». En revanche, le procès envisagé ne devait pas concerner la préparation et la conduite de la guerre par l'Allemagne nazie qui relevait, selon les diplomates du Quai d'Orsay, de la sphère politique et n'était pas à juger dans le cadre d'une telle procédure pénale. Pour le gouvernement français, c'était une considération d'importance cruciale et, par la suite, son point de vue sur cette question l'éloigna de plus en plus de ses Alliés. François de Menthon, alors ministre de la Justice, et qui représenta plus tard la France comme procureur en chef à Nuremberg, déclara en mai 1945 sans équivoque:

«La responsabilité des grands coupables en ce qui concerne le déclenchement même de la guerre en violation des traités et l'établissement de leur politique de guerre ne devrait pas être confondue avec celle des crimes de guerre proprement dits; il s'agit d'une question différente et d'une responsabilité politique ${ }^{(10)}$.

Les Alliés, en particulier du côté américain, ne partageaient pas cette opinion. En effet, l'objectif de ces derniers, en prenant l'initiative de proposer une coopération des quatre grandes puissances alliées principales pour la création d'un tribunal international, était justement de s'en servir pour inculper les dirigeants nazis d'avoir planifié et mené une guerre illégale en violation du droit international. Lors de la conférence de San Francisco (25 avril-26 juin 1945), le plénipotentiaire américain Samuel Rosenman, conseiller du président Truman, fit même comprendre aux ministres des Affaires

8 «Document $\mathrm{n}^{\circ}$ 110: note du Secrétariat des Conférences. Instructions sollicitées par M. Cassin, 16 février 1945 », in: Ministère des AfFaires Étrangères (éd.), Documents diplomatiques français, 1945-1946, t. II, Paris, Imprimerie nationale, 1998, p. 216-218.

9 Ibid., p. 216.

10 «Document no 325: note du Secrétariat des Conférences. Criminels de guerre, 7 mai 1945», in: Ministère des Affaires étrangères (éd.), Documents diplomatiques français, 1945-1946, t. I, Paris, Imprimerie nationale, 1998, p. 606 sq., ici p. 607. Georges Bidault, ministre des Affaires étrangères, s'exprima dans le même sens, voir Archives diplomatiques (AD), NUOI 1944-1959/83, télégramme $\mathrm{n}^{\circ}$ 235-238, 14 mai 1945. 
étrangères britannique, soviétique et français que, pour son gouvernement, c'était même le but principal du procès envisagé contre les grands criminels de guerre ${ }^{(11)}$. Par conséquent, le plan présenté par Rosenman provoqua de fortes tensions franco-américaines au sein du comité qui fut créé à San Francisco pour examiner la proposition des États-Unis et pour élaborer un accord politique des quatre puissances. Le juriste Jules Basdevant, plénipotentiaire du gouvernement français dans cet organisme, informa le Quai d'Orsay «de grandes difficultés» ${ }^{(12)}$ qui auraient surgi dans le débat sur la pénalisation de la guerre d'agression. Mais la poursuite des négociations qui furent, par manque de temps, suspendues à San Francisco et reprises quelques semaines plus tard à Londres montra vite que les Américains restèrent fermes sur leurs intentions et ne cédèrent pas aux demandes françaises. Du côté français, ce conflit eut des conséquences graves: contrarié et déçu par l'échec de ses propres initiatives, Basdevant décida de se retirer de l'affaire et de ne plus participer aux pourparlers. Avant leur reprise à Londres, le ministre des Affaires étrangères, qui appréciait le jugement du juriste, fit de même: convaincu alors que le procès planifié serait "mal engagé» ${ }^{(13)}$, le Quai d'Orsay adopta dès lors "une attitude négative de désintéressement " ${ }^{(14)}$ et ne voulut plus soutenir la création du tribunal international. Désormais, il laissa au ministère de la Justice le soin de suivre les négociations et d'y participer au nom du gouvernement français.

Cependant, le ministère de la Justice n'avait reçu jusque-là que peu d'informations sur le projet et le nouveau garde des Sceaux, Pierre-Henri Teitgen, ayant visiblement d'autres priorités, hésita à en assumer la responsabilité( ${ }^{(15)}$. Dans les négociations qui suivirent, la position française fut fortement fragilisée par l'échec de cette délégation de compétences et, au moment crucial où il fut question d'élaborer le statut du tribunal international et de définir les crimes à punir devant la nouvelle juridiction, l'impression d'une irrésolution et d'une indifférence du gouvernement français s'installa chez les Alliés. En effet, bien que l'ambassade américaine soit intervenue à plusieurs reprises pour rappeler au gouvernement français l'urgence de l'affaire, Teitgen ne réussit pas, des semaines durant, à désigner un nouveau représentant pour les négociations $^{(16)}$. Sous la pression, on choisit finalement au ministère de la Justice le juge Robert Falco, conseiller à la Cour de Cassation, qui fut envoyé à Londres où la conférence des quatre puissances commença le 26 juin $1945^{(17)}$. L'impréparation française, causée par la négligence du cabinet du garde des Sceaux, se manifesta ainsi devant l'ensemble des délégations alliées: en tant que conseiller à la Cour de Cassation, Falco n'était pas en mesure de mener des négociations d'égal à égal avec des personnalités de plus haut rang comme Robert Jackson et David Maxwell Fyfe qui avaient tous les deux

11 AD, NUOI 1944-1959/83, télégramme de Bidault, 6 mai 1945.

12 AD, NUOI 1944-1959/83, crimes de guerre (sans auteur), 14 mai 1945, p. 2.

13 Ibid.

14 AD, NUOI 1944-1959/86, note du Secrétariat des Conférences. Procès de Nuremberg, 28 décembre 1945.

15 Archives nationales (AN), BB 30/1794, Secrétariat des Conférences. Crimes de guerre, 8 mai 1945.

16 AD, NUOI 1944-1959/86, note de l'ambassade des États-Unis, nº 496, 21 mai 1945.

17 Robert Falco, Juge à Nuremberg. Souvenirs inédits du procès des criminels nazis (1945-1946), illustrations de Jeanne Falco, Nancy, Arbre bleu éditions, 2012, p. 28 sq. 
appartenu aux gouvernements de leurs pays et avaient exercé les fonctions d'Attorney General. De plus, Falco arriva seul à Londres tandis que Jackson, par exemple, était accompagné d'une équipe de 16 collaborateurs ${ }^{(18)}$.

Mais la position de la France dans les négociations de Londres fut surtout affaiblie par le fait que Falco n'avait reçu aucune instruction précise de la part du ministère de la Justice lors de son départ. René Massigli, ambassadeur français à Londres, se plaignit pour cette raison de «la complète impréparation de la conférence en ce qui concerne les services de Paris ${ }^{(19)}$. Secondé par André Gros, le délégué adjoint de la France au sein de la Commission des crimes de guerre des Nations unies, et siégeant également dans la capitale britannique, Falco ne put exercer par la suite que peu d'influence sur le déroulement des négociations. Pendant toute la conférence, qui s'acheva le 8 août 1945 avec la signature du statut du TMI, Gros et lui intervinrent rarement et sur presque toutes les questions discutées ils se déclarèrent d'accord avec les propositions anglo-américaines, si bien que les conflits les plus violents qui marquèrent les négociations éclatèrent suite à l'opposition soviétique aux plans de Jackson, sans l'implication des délégués français. Une seule tentative sérieuse de défendre la position française divergeant de celle des Anglo-Américains fut faite par Gros et Falco lorsque le débat porta, une fois encore, sur la création d'un chef d'accusation criminalisant la préparation et la conduite d'une guerre d'agression pour le procès à venir. Ici, les deux délégués eurent recours à la plupart des arguments qui avaient déjà été avancés à San Francisco. Mais les deux Français durent vite constater que sur ce point ils se trouvaient isolés parmi les négociateurs réunis à Londres et que ni les Anglo-Américains ni les Soviétiques ne soutenaient leurs objections fondamentales. $\mathrm{Vu}$ les rapports de forces, ils s'inclinèrent finalement devant la volonté de la majorité et acceptèrent que ce qu'on qualifiait désormais de «crime contre la paix» soit inscrit dans le statut du TMI ${ }^{(20)}$. Au final, le seul succès diplomatique pour la France consista dans le fait même d'être présente à la conférence de Londres, d'y participer et d'être reconnue comme partenaire par les autres puissances alliées. En effet, les délégués français furent loin d'imposer leurs idées ou de participer activement à l'élaboration des bases juridiques du procès de Nuremberg.

\section{La délégation française à Nuremberg}

Le 18 octobre 1945, Charles de Gaulle signa l'ordonnance par laquelle il créa une délégation française auprès du TMI et chargea celle-ci de représenter l'accusation au nom de la France dans le procès des grands criminels de guerre ${ }^{(21)}$. Dans un décret

18 Telford TAYlor, Die Nürnberger Prozesse. Hintergründe, Analysen und Erkenntnisse aus heutiger Sicht, Munich, Heyne, 1994, p. 77.

19 AD, NUOI 1944-1959/83, Massigli à Bidault, 2 juillet 1945.

20 «LII. Revised Definition of "Crimes”, Prepared by British Delegation and Accepted by French Delegation, 28 juillet 1945 », in: United States Department of State (éd.), Report of Robert H. Jackson, United States Representative to the International Conference on Military Trials London 1945, Washington, U.S. Government Printing Office, 1949, p. 390 sq.

21 «Ordonnance n 45-2397 du 18 octobre 1945 instituant une délégation du Gouvernement provisoire de la République française au ministère public du tribunal militaire international», JORF, 19 octobre 1945, p. 6652. 
qui suivit le même jour, la composition et le fonctionnement de la délégation furent réglés ${ }^{(22)}$. Les effectifs de la délégation varièrent pendant les mois qui suivirent: en novembre 1945, quand le procès des grands criminels de guerre débuta, l'effectif total de la délégation française était de 62 personnes ${ }^{(23)}$. Jusqu'à la fin de l'année, un grand nombre de recrutements suivirent, si bien que la délégation s'agrandit considérablement $^{(24)}$ et atteignit sa taille maximale de 100 employés début janvier $1946^{(25)}$. Mais il est à noter qu'on ne peut parler ici que de la délégation au sens strict. Il s'agissait de toutes les personnes qui participaient directement au procès et qui avaient le statut de salariés du ministère de la Justice: les juges, les procureurs, leurs assistants, les traducteurs et interprètes, les secrétaires et sténotypistes. Les nombres mentionnés n'incluent pas les personnes qui séjournaient à Nuremberg par ordre d'autres ministères et services, mais qui, au sens large, appartenaient également à la délégation française. Il s'agissait surtout d'un détachement militaire déployé à Nuremberg pour garantir la sécurité de la délégation française qui comptait 85 soldats en janvier $1946^{(26)}$. Une autre équipe fut envoyée par les PTT pour assurer une communication par téléphone et télégraphe avec Paris ${ }^{(27)}$. En outre, des représentants des ministères des Affaires étrangères et de l'Information arrivèrent également à Nuremberg avec leurs propres missions. En janvier 1946, François de Menthon, comme il l'écrivit dans une lettre à de Gaulle, avait au total 262 personnes sous sa direction ${ }^{(28)}$. Avec un tel effectif la délégation française était, après l'américaine, la deuxième plus grande représentation alliée auprès du $\mathrm{TMI}^{(29)}$.

Le personnel juridique, qui effectuait le travail au sein même du procès, était composé des membres du tribunal et du ministère public. Les deux juges français du TMI furent Henri Donnedieu de Vabres, professeur de droit criminel à la faculté de droit de Paris, et Robert Falco qui avait représenté la France à la conférence de Londres. Du côté du ministère public il y avait une hiérarchie à quatre niveaux: en haut de cette hiérarchie se trouvait le poste de procureur en chef. Ce dernier fut occupé d'abord par François de Menthon qui démissionna en janvier 1946, puis par son successeur

22 «Décret n 45-2415 du 18 octobre 1945 fixant la composition et le fonctionnement de la délégation du Gouvernement provisoire de la République française au ministère public du tribunal militaire international», JORF, 19 octobre 1945, p. 6666.

23 AN, 19970410/1, liste des membres et employés auxiliaires, 27 novembre 1945; voir également Dominique Tantin (éd.), Delphin Debenest, 1939-1945. Un magistrat français en guerre contre le nazisme, La Crèche, Geste, 2005, p. 290.

24 «Délégation du Gouvernement provisoire de la République française au tribunal militaire international», JORF, 18 novembre 1945, p. 7663 sq.; voir également JORF, 19 décembre 1945, p. 8390.

25 AN, 19970410/1, état de présence des membres de la délégation française au siège du Tribunal Militaire International, Nuremberg, janvier 1946.

26 AD, HCRFA/SL/19, le Général de Corps d’Armée Koenig, Commandant en chef français en Allemagne. Détachement français de Garde auprès du Tribunal Militaire International de Nuremberg, 16 octobre 1946; AN, F 41/2721, de Menthon à de Gaulle, 19 janvier 1946, p. 4.

27 «Le procès de Nuremberg ne sera plus remis», Le Parisien libéré, 6 novembre 1945.

28 AN, F 41/2721, de Menthon à de Gaulle, 19 janvier 1946, p. 4.

29 Ann Tusa, John Tusa, The Nuremberg Trial, New York, Cooper Square Press, 2003, p. 136; T. TAY LOR, Die Nürnberger Prozesse (note 18), p. 256; (David Maxwell Fy fE), Political Adventure. The Memoirs of the Earl Kilmuir, Londres et al., Weidenfeld \& Nicolson, 1964, p. 103. 
Auguste Champetier de Ribes ${ }^{(30)}$. Viennent ensuite les deux postes d'adjoint du procureur qui furent occupés par Charles Dubost et, pendant une longue phase du procès, Edgar Faure, qui n'était alors qu'au début de sa carrière politique ${ }^{(31)}$. Le troisième niveau de cette hiérarchie se composait des chefs de section auquel appartenaient notamment Delphin Debenest, Charles Gerthoffer, Jean Leyris et Pierre Mounier ${ }^{(32)}$. Ils étaient quant à eux responsables des différents thèmes que couvrait l'accusation contre les dirigeants nazis. Sous leur direction travaillaient enfin plusieurs chargés de mission qui complétaient le ministère public et qui préparaient les exposés de l'accusation française. Ces fonctions furent remplies par Aline Chalufour, Henri Delpech, Serge Fuster, Jacques-Bernard Herzog, Jean-Jacques Lanoire, Henri Monneray et Constant Quatre.

De par leurs biographies, les membres du ministère public formaient un groupe assez homogène. À Nuremberg, il s'agissait de dénoncer les crimes du nazisme et de demander justice au nom de la France meurtrie par l'occupant allemand. Une telle accusation ne pouvait être portée de manière crédible et convaincante que par des procureurs n'ayant pas été compromis personnellement durant la guerre. C'est pourquoi le ministère de la Justice choisit des magistrats ayant personnellement contribué au combat contre l'Allemagne nazie et qu'on ne pouvait pas soupçonner d'avoir été complices des occupants ou des sympathisants du régime de Vichy. On composa ainsi un parquet dont pratiquement tous les membres avaient été des résistants éprouvés. Charles Dubost, qui avait été fait prisonnier par les Allemands, réussit à s'enfuir pendant la guerre. En tant que substitut du parquet de Toulon, il organisa ensuite le transport des armes pour le mouvement Libération, devint membre d'un réseau d'exfiltration vers l'Algérie, hébergea chez lui des officiers alliés ayant débarqué en secret dans la France occupée, établit des dépôts de matériel de guerre et se rallia en été 1944 aux FFI ${ }^{(33)}$. Jean Leyris, en tant que président du Tribunal civil de Carpentras, avait soutenu un groupe qui commit de nombreux actes de sabotage contre les forces d'occupation $^{(34)}$. Après une spectaculaire attaque à l'explosif, au cours de laquelle une vingtaine de locomotives du dépôt ferroviaire d'Avignon furent détruites, il fut arrêté en avril 1944 par la police allemande. Quelques semaines plus tard, il réussit à s'échapper d'un convoi de déportation en route vers Dachau.

Pierre Mounier fut victime des mesures d'épuration prises par le régime de Vichy ${ }^{(35)}$. À cause de son appartenance à une loge maçonnique, il fut exclu du service public en 1941 et perdit son poste de juge à la Cour d'appel de Rabat. Après le débarquement allié au Maroc, il rejoignit les troupes gaullistes et rentra en France comme inspecteur de la justice militaire dans les territoires libérés. Henri Monneray, né sous le nom de Heinz

30 AN, 19970410/1, note sur la composition de la délégation française près le TMI, 3 juillet 1947. Sur de Menthon et Champetier de Ribes, voir Laurent Ducerf, François de Menthon. Un catholique au service de la République (1900-1984), Paris, Éditions du Cerf, 2006; Philippe Dazet-Brun, Auguste Champetier de Ribes (1882-1947). Un catholique social en République, Biarritz, Séguier, 2008.

31 Faure consacra une partie de ses mémoires à son séjour à Nuremberg; Edgar FAure, Mémoires II: «Si tel doit être mon destin ce soir », Paris, Plon, 1984, p. 11-64.

32 AN, BB-30-1779, liste sans date: «Tribunal Militaire International, ministère public».

33 AN, 19890322/43, dossier de carrière: Dubost, Charles Joseph Marie.

34 AN, 19840317/7, dossier de carrière: Leyris, Jean Marius Joseph.

35 AN, 19770067/338, dossier de carrière: Mounier, Pierre Édouard François. 
Meierhof à Erfurt, avait vécu en France occupée, en tant que Juif, dans l'illégalité(36). À Tarbes, il joua un rôle actif dans une filière de passeurs conduisant des aviateurs alliés vers l'Afrique du Nord. Jacques-Bernard Herzog s'était engagé pendant la guerre dans un mouvement de résistance à Toulouse ${ }^{(37)}$. Craignant son arrestation, il décida de fuir vers l'Espagne en plein hiver 1943. Dans le froid des Pyrénées, il souffrit de plusieurs gelures qui nécessitèrent l'amputation de son pied gauche. Delphin Debenest avait également payé cher son courage: arrêté en tant que résistant en juillet 1944 à Poitiers, il fut déporté au camp de concentration de Buchenwald et ne rentra en France qu'à la fin de la guerre ${ }^{(38)}$.

De tels mérites ne pouvaient pas être mis en doute et expliquèrent la grande majorité des nominations. Mais on savait bien au ministère de la Justice que la réalité des années de guerre avait été plus complexe et que surtout les magistrats s'étaient alors retrouvés dans une situation bien difficile ${ }^{(39)}$. En tant que membre d'un système étatique qui les obligeait à se soumettre aux lois et aux ordres hiérarchiques, les magistrats devaient sous Vichy servir le régime s'ils voulaient continuer à exercer leur fonction et garder leurs postes ${ }^{(40)}$. La «Résistance judiciaire» se caractérise donc surtout par sa complexité: toutes les activités résistantes, dont les formes et l'intensité avaient varié considérablement au sein de la magistrature, nécessitaient le couvert de la loyauté professionnelle au régime ${ }^{(41)}$. C'est pourquoi la plupart des membres du parquet français avaient prêté serment de fidélité à la personne du maréchal Pétain, comme cela était exigé des magistrats sous le régime de Vichy ${ }^{(42)}$. Quelques-uns étaient même allés plus loin dans leurs manifestations de loyauté envers le régime: après avoir été démobilisé, Serge Fuster se rendit en été 1940 à Vichy où il se présenta à plusieurs reprises, avec une rare opiniâtreté, au ministère de la Justice qui y était installé depuis peu. C’est ainsi qu'il réussit à être intégré dans la magistrature. Pour, semble-t-il, réfuter des accusations sur ses convictions politiques, Pierre Mounier adhéra quelques mois avant son expulsion de la magistrature à la Légion française des combattants (LFC), créée par le régime de Vichy, et souligna dans une lettre datée de juillet 1941 «l'admiration, le respect et la reconnaissance» ${ }^{(43)}$ qu'il éprouvait pour le maréchal Pétain. Même à propos de Jean Leyris ses supérieurs à la Cour d'appel de Nîmes notèrent en novembre 1943: «il est sincèrement dévoué aux principes de l’ordre nouveau et fidèle

36 AN, 19950468/4, dossier: Monneray, Henri.

37 AN, 19890074/124, dossier de carrière: Herzog, Jacques Bernard.

38 AN, 19820583/146, dossier de carrière: Debenest, Delphin Jean Aristide.

39 Sur la magistrature sous Vichy, voir Alain BANCAUD, Une exception ordinaire. La magistrature en France 1930-1950, Paris, Gallimard, 2002.

40 Ibid., p. 444.

41 Sur la «Résistance judiciaire», voir Liora IsRAëL, Robes noires, années sombres. Avocats et magistrats en résistance pendant la Seconde Guerre mondiale, Paris, Fayard, 2005; Liora IsRAëL, «Résister par le droit? Avocats et magistrats dans la résistance (1940-1944)», L’Année sociologique, 59/1 (2009), p. 149-175.

42 Par exemple AN, 19890074/132, dossier de carrière: Lanoire, Jean Jacques Joseph, «Prestation de serment ", 4 novembre 1942.

43 AN, 19770067/338, dossier de carrière: Mounier, Pierre Édouard François, lettre manuscrite de Pierre Mounier du 22 juin 1941. 
à la personne du Chef de l'État ${ }^{(44)}$. Sous Vichy tous les magistrats devaient, d'une manière ou d'une autre, s'arranger avec le régime et le ministère de la Justice en était bien conscient en 1945 .

C'est pourquoi l'éventuelle loyauté manifestée envers Vichy par un candidat au TMI ne constitua pas en tant que telle un obstacle pour son recrutement. Mais ce que les responsables au ministère de la Justice craignaient vraiment et cherchaient à éviter à tout prix étaient des scandales publics qui pouvaient éclater à propos de leurs décisions et qui pouvaient nuire à l'image de la France. Quand un tel danger apparaissait, le ministère réagissait rapidement et avec la plus grande fermeté. Henri Delpech fut confronté à cette fermeté après avoir présenté en janvier 1946 un exposé au procès de Nuremberg, dont rendit compte la presse française ${ }^{(45)}$. Le lendemain, d'anciens soldats ayant été détenus avec lui en Allemagne s'adressèrent au ministère de la Justice pour déclarer que Delpech avait appartenu en captivité à un cercle pétainiste. La réaction du ministère, qui redoutait un scandale au cas où les accusations contre Delpech seraient relayées par les médias, est révélatrice: Delpech dut rentrer à Paris, où il fut informé de son expulsion immédiate de la délégation ${ }^{(46)}$. Cette affaire montre bien dans quelle mesure les responsables au ministère de la Justice étaient conscients du caractère très sensible du choix du personnel pour le procès de Nuremberg et leurs décisions dépendaient grandement de considérations politiques.

\section{L'accusation française}

Dans la salle d'audience du palais de justice de Nuremberg, l'accusation française eut la parole du 17 janvier au 7 février 1946 pour présenter, preuves à l'appui, ses inculpations contre les dirigeants nazis. Devant les autres participants au procès, mais aussi devant un grand public de journalistes venus du monde entier, elle pouvait alors exposer son point de vue sur les crimes allemands de la Seconde Guerre mondiale et les conclusions à en tirer. En partie, ce point de vue différait considérablement de ce que les procureurs anglo-américains, mais aussi soviétiques, affirmèrent.

C'était surtout son positionnement sur la question de la culpabilité collective des Allemands qui sépara le parquet français des Alliés en donnant la responsabilité générale à l'ensemble du peuple allemand. Pour la première fois, cette thèse fut défendue au procès de Nuremberg par François de Menthon dans le discours d'ouverture qu'il présenta au nom de sa délégation ${ }^{(47)}$. À partir de ce moment-là, elle devint un leitmotiv de toute l'accusation française régulièrement répété par les compatriotes de l'ancien

44 AN, 19840317/7, dossier de carrière: Leyris, Jean Marius Joseph, Cour d'Appel de Nîmes, notice du Président et du Procureur: «Leyris, Jean», 19 novembre 1943.

45 Der Prozeß gegen die Hauptkriegsverbrecher vor dem Internationalen Militärgerichtshof, 14. November 1945 bis 1. Oktober 1946, amtlicher Text in deutscher Sprache, 42 vol., Nuremberg, 1947-1949, ici vol. 5, p. 633-644 et vol. 6, p. 7-28.

46 AN, 19890322/39, dossier de carrière: Delpech, Henri Joseph Jules Léon.

47 Annette Weinke, Die Nürnberger Prozesse, Munich, Beck, 2006, p. 41; Matthias GemäHLICH, «Wir aber wollen, dass dieses Deutschland schuldig gesprochen werde - Zur Eröffnungsrede des französischen Chefanklägers François de Menthon », in: Nürn berger Menschenrechtszentrum (éd.), Das Internationale Militärtribunal von Nürnberg 1945/1946. Die Reden der Hauptankläger neu gelesen und kommentiert, Hambourg, CEP Europäische Verlagsanstalt, 2015, p. 127-162, ici p. 137-140. 
garde des Sceaux, ce qui frappa les auditeurs. Viktor von der Lippe, un des avocats allemands, nota lors d'une phase ultérieure du procès dans son journal:

«En utilisant sans cesse des formulations généralisantes, les procureurs français tendent nettement à déclarer le peuple allemand entier comme coupable. À cet égard, ils vont beaucoup plus loin que les Anglo-Saxons » ${ }^{(48)}$.

Mais ce n'était pas le seul cas où les procureurs français se démarquaient des Alliés: l'inculpation contre les dirigeants nazis d'avoir planifié et mené une guerre d'agression à laquelle les délégations anglo-américaines, mais aussi soviétique attachaient beaucoup d'importance manque presque entièrement dans l'accusation française. Évidemment, les différends entre les Alliés, nés au moment de la phase de préparation du procès, quand les représentants français avaient en vain essayé d'écarter du procès le déclenchement de la guerre comme chef d'accusation, se poursuivaient ici et le parquet français, soucieux de montrer un front uni avec les autres délégations, n’inséra dans son discours d'ouverture qu'un bref aperçu rédigé par Pierre Renouvin qui défendait la thèse de la criminalité de la guerre d'agression devant le droit international ${ }^{(49)}$. En revanche, les grands sujets sur lesquels l'accusation française se concentrait étaient le travail forcé, le pillage économique, les atrocités sous toutes les formes et la politique de germanisation dans les territoires occupés. Cherchant visiblement à fonder leurs inculpations sur un droit positif, les procureurs français se référaient à des traités internationaux, surtout à la convention de La Haye de 1907, mais aussi à la législation nationale des pays concernés pour démontrer la criminalité des faits en question. À la fin du procès, il s'avéra que ce procédé fut couronné de succès: le tribunal accepta presque complètement l'argumentation française relative aux crimes de guerre et souligna dans son jugement l'illégalité du travail forcé et du pillage économique pratiqués par l'Allemagne nazie. Les atrocités allemandes dans les pays d'Europe de l'Ouest, mais aussi dans les camps de concentration que l'accusation française avait fustigées, furent également prises en compte par les juges ${ }^{(50)}$. Dans le jugement, ils mentionnèrent par exemple explicitement le massacre d'Oradour-sur-Glane auquel Charles Dubost avait consacré une partie considérable de son temps de parole devant le tribunal ${ }^{(51)}$.

C'est seulement sur la question des exécutions d'otages, qu'elle aurait a priori voulu voir qualifiées de crimes de guerre, que l'accusation française ne réussit pas à s'impo$\operatorname{ser}^{(52)}$. Ici, le tribunal évita une prise de position de principe et se borna dans son jugement au simple constat qu'un grand nombre d'otages avait été fusillés par les troupes allemandes sans faire de déclaration supplémentaire sur l'illégalité de ces représailles. En outre, l'interprétation française du crime contre l'humanité ne convainquit pas le tribunal: l'accumulation des inculpations d'une nature très diverse qu'Edgar Faure

48 Viktor Freiherr von der Lippe, Nürnberger Tagebuchnotizen. November 1945 bis Oktober 1946, Francfort-sur-le-Main, Knapp, 1951, p. 112 (citation traduite par l'auteur). Ibid., p. 262. Sur l'argumentation française dans ce cas, voir Peter Lieb, Konventioneller Krieg oder NS-Weltanschauungskrieg? Kriegführung und Partisanenbekämpfung in Frankreich 1943/44, Munich, Oldenbourg, 2007, p. 254 sq. 
présenta au tribunal sous ce titre et qu'il qualifia de politique criminelle visant à germaniser les territoires occupés fut presque entièrement laissée de côté par les juges du TMI. L'incorporation de force que les Allemands avaient pratiquée en Alsace et en Moselle, par exemple, ne fut même pas mentionnée dans le jugement ${ }^{(53)}$. La seule partie des inculpations présentées par Faure que le tribunal entérina et mentionna dans son jugement était la persécution des Juifs en France et dans les autres pays de l'Europe de l'Ouest ${ }^{(54)}$. Les réserves du tribunal, en ce qui concerne cette question, étaient dues au fait que l'interprétation française du crime contre l'humanité, comme elle fut développée pendant le procès, dépassait largement les termes du statut de Londres qui contenait une définition étroite et qui liait les crimes contre l'humanité aux autres chefs d'accusation. Les juges du TMI appliquèrent cette disposition rigoureusement et ne distinguèrent pas clairement dans leur jugement les crimes contre l'humanité des crimes de guerre, ce qui ne laissa pas de place pour la conception française ${ }^{(55)}$.

On peut constater une autre spécificité française lorsqu'on porte son regard sur les qualifications juridiques des inculpations présentées devant le TMI. Le parquet français ne définit et ne catégorisa les innombrables crimes allemands qu'en fonction de la chronologie et des méthodes employées. En revanche, il y manqua une distinction explicite entre les différents groupes de victimes. Par exemple, les procureurs a priori ne différencièrent pas dans leurs exposés devant le tribunal les Juifs systématiquement exterminés dans le cadre de la Shoah et les résistants torturés et assassinés dans le cadre du combat que les occupants menèrent contre la Résistance. Les déportations «raciales» et politiques furent considérées par eux comme des crimes de même nature, perpétrés pour les mêmes mobiles ${ }^{(56)}$. Dubost, qui présenta cette partie de l'accusation française, ne mentionna souvent qu'en marge l'identité juive des victimes ${ }^{(57)}$. Une telle généralisation amena quelques historiens à reprocher au parquet français de Nuremberg de ne pas avoir suffisamment traité de l'extermination des Juifs ou même de s'être tu sur ce sujet pour cacher la complicité du régime de Vichy dans les déportations ${ }^{(58)}$. Lorsqu'on examine de plus près les arguments de l'accusation française, de tels reproches s'avèrent injustifiés. En fin de compte, une grande partie des exposés fut consacrée aux crimes perpétrés à l'encontre des Juifs et la délégation mena une

53 Frédéric Stroh, Introduction, in: Frédéric Stroh, Peter M. QuAdFlieg (éd.), L'incorporation de force dans les territoires annexés par le III Reich 1939-1945, Strasbourg, Presses universitaires de Strasbourg, 2016, p. 5-25, ici p. 8.

54 Der Prozeß gegen die Hauptkriegsverbrecher (note 45), vol. 1, Urteil, p. 280 sq.

55 Ibid., p. 285 sq.; voir aussi Rainer Huhle, "Vom schwierigen Umgang mit "Verbrechen gegen die Menschheit" in Nürnberg und danach», s.l., 2009. Disponible sur: www.menschenrechte.org/ blog/2009/02/17/verbrechen-gegen-die-menschheit/, p. 4 sq.; Mireille Delmas-Marty, Isabelle FouChard, Emanuela Fronza, Laurent Neyret, Le crime contre l'humanité, Paris, Presses universitaires de France, 2009, p. 14 sq.

56 Der Prozeß gegen die Hauptkriegsverbrecher (note 45), vol. 5, p. 452 sq.

57 Ibid., vol. 6, p. 175 sq.

58 Donald Bloxнам, Genocide on Trial. War Crimes Trials and the Formation of Holocaust History and Memory, Oxford et al., Oxford University Press, 2003, p. 101 sq.; Henry Rousso, «Juger le passé? Justice et histoire en France», in: Florent BrayARD (éd.), Le Génocide des Juifs entre procès et histoire 1943-2000, Actes d'un colloque, Berlin et Potsdam janvier 1998, Bruxelles, Complexe, 2000, p. 261-287, ici p. 270; A. WeInke, Die Nürnberger Prozesse (note 47), p. 49. 
coopération étroite avec le Centre de documentation juive contemporaine (CDJC) à Paris qui mit à la disposition des juristes un grand nombre de documents produits par la suite comme preuves à Nuremberg ${ }^{(59)}$.

Dans sa méthodologie, l'accusation française différait également d'une manière évidente des présentations des autres délégations alliées, notamment de celles des Anglo-Américains. Tandis que les parquets américain et britannique produisaient principalement comme preuves des documents allemands, confisqués par leurs troupes et axés sur les accusés et leurs mobiles, l'accusation française quant à elle adoptait la perspective des victimes ${ }^{(60)}$. Dubost, par exemple, développa ses inculpations sur la base d'un grand nombre de rapports policiers français et de témoignages écrits qui décrivaient des atrocités allemandes commises partout en France. Il argumenta que l'ampleur de ces crimes ne pouvait s'expliquer que par une politique bien réfléchie et dirigée par les accusés qui avaient occupé les postes les plus hauts dans le gouvernement, le parti nazi et l'armée.

Cette argumentation devait certainement avoir pour but d'une part de masquer le point faible de l'accusation française: en effet, contrairement aux Anglo-Américains, le parquet français ne disposait que de peu de documents allemands qui prouvaient quels ordres avaient été signés et quelles décisions avaient été prises individuellement par chaque accusé. Mais d'autre part, elle reflétait aussi une autre stratégie pour le procès et une autre compréhension de son but. L'intention déclarée du parquet français était de tourner l'attention générale vers les victimes et de leur donner la parole devant le tribunal. Pour cette raison, dans le cadre de l'accusation française, furent interrogés onze témoins qui appartenaient tous au groupe des victimes du nazisme. Dubost, pour qui ces dépositions étaient d'une importance de tout premier plan, leur réserva entièrement deux audiences ${ }^{(61)}$. Les témoins français furent choisis par la délégation en coopération avec la Fédération nationale des déportés et internés résistants et patriotes (FNDIRP) et étaient censés créer, par leurs dépositions, une forte impression au tribunal, mais aussi aux observateurs du procès ${ }^{(62)}$. Dans un procès qui était dans l'ensemble centré sur les dirigeants nazis, le mérite revint alors à la délégation française d'avoir aussi fait entendre la voix des victimes ${ }^{(63)}$.

59 A. Tisseron, La France et le procès de Nuremberg (note 6), p. 153 sq.

60 Sur la stratégie des parquets américain et anglais, voir Tusa/Tusa, The Nuremberg Trial (note 29), p. 101.

61 Der Prozeß gegen die Hauptkriegsverbrecher (note 45), vol. 6, p. 359.

62 La coopération avec la FNDIRP explique aussi pourquoi des rescapés des camps de Buchenwald et Mauthausen surtout déposèrent pour le parquet français à Nuremberg: les associations de déportés avaient leurs origines dans les camps avec un grand nombre de détenus français qui y avaient formé les premiers réseaux, surtout à Buchenwald et Mauthausen; Serge Wolikow, Les combats de la mémoire. La FNDIRP de 1945 à nos jours, Paris, le Cherche-Midi, 2006, p. 27-61 ; aussi Michel FABrÉGuet, Mauthausen. Camp de concentration national-socialiste en Autriche rattachée (1938-1945), Paris, Honoré Champion, 1999, p. 573-585; Olivier LALieu, La zone grise? La Résistance française à Buchenwald, Paris, Tallandier, 2005.

63 Le contraire se passa en 1961 au procès contre Adolf Eichmann à Jérusalem où le parquet cita un grand nombre de rescapés juifs des camps de concentration à la barre et leur donna la parole; Annette WIEviorka, L’ère du témoin, Paris, Fayard, 2013; Annette Wieviorka, Le procès Eichmann, Bruxelles, Complexe, 1989. 
Lorsqu'on essaie d'évaluer l'accusation française à Nuremberg au regard de l'état des recherches d'aujourd'hui, il faut tout d'abord rappeler qu'en 1945, peu de mois après la fin de la guerre, les connaissances exactes sur les crimes allemands étaient toujours limitées. Ainsi il n'est pas surprenant que plusieurs données chiffrées, avancées par l'accusation française devant le TMI, durent être corrigées. Dubost parla au procès de Nuremberg de 250000 personnes qui furent déportées de France dans des camps de concentration et des prisons allemandes ${ }^{(64)}$. Aujourd'hui, on chiffre le nombre des déportés de France à $135000^{(65)}$. Dans le cas des exécutions d'otages ce n'est pas que le nombre de 29660 victimes soit exagéré, mais Dubost s'est appuyé sur une définition floue. En effet, d'après une statistique du Service de recherche des crimes de guerre ennemis (SRCGE) qu'il présenta comme pièce à conviction, il considéra toutes les personnes tuées par les occupants comme otages fusillés, y compris les résistants et maquisards morts au combat ${ }^{(66)}$. Aujourd'hui, on estime que le nombre total des personnes tuées par les occupants en France est de 20000 personnes, dont 5000 étaient des civils. À peu près 1000 personnes furent exécutées comme otages au sens propre ${ }^{(67)}$.

Mais il y avait aussi des données chiffrées dans les exposés de l'accusation française qui s'avérèrent assez exactes et qui furent plus tard confirmées par les historiens: Jacques-Bernard Herzog, par exemple, parla devant le TMI de 2600000 Français forcés à travailler pour l'occupant, dont 876000 auraient été déportés en Allemagne, et donna ainsi une image réaliste des dimensions de la politique nazie du travail forcé(68). C'est pourquoi, on peut constater que la délégation française a dans l'ensemble réussi à accomplir sa mission bien qu'elle ait travaillé sous la pression permanente et le manque de temps, qu'elle ait dû s'habituer à une procédure judiciaire inconnue et qu'elle n'ait disposé que de ressources restreintes. Elle produisit des preuves pertinentes devant le TMI et en tira des conclusions convaincantes, comme le montre la confirmation de la plupart des inculpations françaises dans le verdict à la fin du procès. À juste titre François de Menthon parla plus tard d'une "contribution très utile à l'accusation » ${ }^{(69)}$ faite par lui-même et son équipe à Nuremberg.

64 Der Prozeß gegen die Hauptkriegsverbrecher (note 45), vol. 6, p. 363 ; Dubost déclara que sur les 250000 à peu près 35000 auraient survécu. Sur ce point-là il fit référence à un rapport du ministère des Prisonniers, Déportés et Réfugiés qu'il présenta sous la cote F-497/RF-399; Centre de documentation juive contemporaine (CDJC), Nuremberg-CCCXCIV/14.

65 Ce chiffre total comprend 75000 déportés juifs et 60000 déportés politiques. Presque la totalité des déportés juifs furent assassinés (2560 rescapés), dans le groupe des déportés politiques à peu près un tiers ne survécut pas à la détention; Pierre LAGrou, «Les guerres, les morts et le deuil. Bilan chiffré de la Seconde Guerre mondiale», in: Stéphane Audoin-Rouzeau, Annette Becker, Christian Ingrao, Henry Rousso (éd.), La violence de guerre 1914-1945, Bruxelles, Complexe, 2002, p. 313-339, ici p. 319 sq.; Corine Defrance, Ulrich Pfeil, Eine Nachkriegsgeschichte in Europa, 1945 bis 1963, Darmstadt, WBG, 2011, p. 22; Serge Klarsfeld, Vichy - Auschwitz. Die "Endlösung der Judenfrage" in Frankreich, Darmstadt, WBG, 2007, p. 368-372.

66 Der Prozeß gegen die Hauptkriegsverbrecher (note 45), vol. 6, p. 154; la liste fut présentée par Dubost sous la cote F-420/RF-266; AN, BB 30/1796, statistique des otages fusillés, fournie par les délégués régionaux du Service de recherche des crimes de guerre, sans date.

67 P. Lieb, Konventioneller Krieg (note 52), p. 412-415.

68 Der Prozeß gegen die Hauptkriegsverbrecher (note 45), vol. 5, p. 567.

69 Archives départementales de la Haute-Savoie (ADHS), 135 J 191, de Menthon, manuscrit, vers 1966, p. 2. 


\section{La perception en France}

Le 2 octobre 1946, il y avait à la une de pratiquement tous les journaux français des gros titres comme «Le verdict de Nuremberg: Douze condamnations à mort par pendaison » ${ }^{(70)}$ ou: «Onze chefs nazis sur vingt-deux seront pendus » ${ }^{(71)}$. Le jugement que le TMI avait rendu à Nuremberg fut plusieurs jours durant un des thèmes principaux de la couverture médiatique en France.

Pourtant, les journaux français étaient loin de faire l'éloge de la tâche accomplie par les juristes des pays alliés et se montrèrent très critiques devant les résultats du procès: dans les médias, on parla de la "honte de Nuremberg " ${ }^{(72)}$ et on fustigea surtout les trois acquittements que le TMI avait rendus à la grande stupéfaction de l'opinion publique française. Les sept peines de prison qui furent prononcées lors du jugement furent également considérées par un grand nombre de journalistes comme trop clémentes. Le cas de Rudolf Heß notamment suscita de vives réactions : l'opinion selon laquelle ce dernier aurait mérité la peine de mort au lieu de la réclusion criminelle à perpétuité était largement répandue parmi les commentateurs. De plus, la décision du TMI de ne pas déclarer criminels la SA, le cabinet du Reich et le haut commandement des forces armées allemandes renforçait l'indignation générale. Cet «acquittement collectif ${ }^{(73)}$ fut interprété par les détracteurs du jugement comme une réhabilitation de l'idéologie nazie. La véhémence de la critique dépendait certes de la position politique de chaque journal. À gauche, une véritable tempête d'indignation se leva: "Outrage à la conscience humaine - Trois des accusés sont acquittés, sept autres échappent à la potence ${ }^{(74)}$, commenta par exemple Le Populaire de Paris et pour L'Humanité le jugement de Nuremberg était "une injure à tous les martyrs de la liberté ${ }^{(75)}$. En évoquant les sacrifices de la Résistance pour justifier son indignation, le quotidien communiste se servit d'un argument sans aucun doute puissant qui trouva un fort écho dans tous les camps politiques. Pour L'Aurore, il s'agissait d'un "verdict qui soulève des protestations » ${ }^{(76)}$, le journal Résistance s'indigna des «scandaleux acquittements " ${ }^{(77)}$ et le quotidien Front national voyait une "vague d'indignation en France» ${ }^{(78)}$ provoquée par les juges du TMI. Dans le Franc-Tireur, un autre quotidien issu de la Résistance, la journaliste Madeleine Jacob qui avait assisté personnellement à un grand nombre d'audiences du TMI à Nuremberg ne cacha pas sa déception et dressa un bilan plein d'amertume:

«Le tribunal international a failli à sa tâche. L'aube qui se lèvera sur le monde de demain est une aube trouble. Supprimer les criminels, c'est bien. Mais ne pas supprimer tous les criminels et leurs instigateurs et leurs soutiens, c'est un crime. L'humanité paiera ${ }^{(79)}$.

Le Monde, 2 octobre 1946.

72 «Après la grande honte de Nuremberg», Front National, 3 octobre 1946.

73 France Libre, $1^{\text {er }}$ octobre 1946.

74 Le Populaire de Paris, 2 octobre 1946.

L'Humanité, 2 octobre 1946.

L'Aurore, 2 octobre 1946.

77 Résistance, 2 octobre 1946.

78 Front National, 3 octobre 1946. 
Parmi les détracteurs les plus violents du jugement de Nuremberg figurait aussi l'écrivain Pierre Schaeffer qui reprocha aux juges du TMI: «Vous êtes des lâches " ${ }^{\left({ }^{(0)}\right.}$ et admit avec une franchise sans équivoque: «Quand la justice est sans proportion avec le crime, je préfère la vengeance ${ }^{(81)}$.

Devant ce tollé médiatique, peu nombreux étaient ceux qui approuvèrent le jugement du TMI ou qui au moins restèrent plus prudents dans leurs commentaires. L'Aube, journal chrétien-démocrate, se borna à constater que le jugement «sera[it] diversement commenté» ${ }^{(82)}$. Le Figaro salua explicitement le "grandiose événement ${ }^{(83)}$ qui se serait déroulé à Nuremberg et pour L'Époque Jean Bernard-Derosne rédigea une chronique dans laquelle il déclara: "Le tribunal a ses raisons. Mais les Français ne trouveront pas, eux, de raisons d'accepter sans passion cette clémence indigeste ${ }^{\left({ }^{84)}\right.}$. Compte tenu de ces hommages, il faut certainement constater que les commentaires des journaux conservateurs et libéraux étaient plus nuancés tandis que les condamnations furent catégoriques et unanimes à gauche. Mais dans l'ensemble, c'était un rejet général que le jugement du TMI trouva dans la presse française et bien peu de commentateurs ne partageaient pas l'indignation de la grande majorité des journalistes.

Les grandes associations des anciens déportés et résistants manifestèrent également leur mécontentement face au jugement. Leurs réactions furent encore plus virulentes que celles de la presse et elles n'hésitèrent pas à lancer une campagne politique en faisant pression sur le Quai d'Orsay pour qu'il intervienne auprès des Alliés contre le jugement. Maurice Lampe, le secrétaire général de la FNDIRP qui avait lui-même déposé en tant que témoin au procès de Nuremberg, estimait nécessaire d'avertir les diplomates que «le maintien de ces verdicts serait un encouragement à la répétition des atrocités du fascisme» ${ }^{(85)}$. C'est pourquoi la FNDIRP exigea que le verdict fût annulé et que tous les accusés fussent de nouveau condamnés - à la peine de mort. De telles revendications furent partagées par d'autres associations et syndicats partout en France: l'Association départementale des prisonniers de guerre du Pas-de-Calais réclama la révision du procès de Nuremberg tout comme l'Association républicaine des anciens combattants à Agen, l'Amicale des prisonniers, déportés et internés de la régie intéressée du gaz de Marseille, la Fédération régionale des mineurs du Gard ou par exemple le Syndicat des cheminots d'Avignon ${ }^{(86)}$.

Dans leurs démarches, les formulations ne pouvaient pas être assez sévères: pour le Syndicat général du papier-carton et similaires des départements de Seine

80 Pierre Schaeffer, «Les voleurs de cadavres», Esprit, 12 (1946), p. 829-840, ici p. 830.

81 Ibid.

82 «Onze chefs nazis sur vingt-deux seront pendus", L’Aube, 2 octobre 1946.

83 «Le Jugement», Le Figaro, 2 octobre 1946.

84 Ibid.

85 AD, NUOI 1944-1959/91, Lampe au nom de la FNDIRP, 7 octobre 1946.

86 AD, NUOI 1944-1959/91, lettres: l'Association départementale des prisonniers de guerre du Pasde-Calais, 17 octobre 1946; l'Association républicaine des anciens combattants de la section d'Agen, 25 octobre 1946; l’Amicale des prisonniers, déportés et internés de la régie intéressée du gaz de Marseille, 3 octobre 1946; la Fédération régionale des mineurs du Gard, 5 octobre 1946; le Syndicat des cheminots Avignon et région, 11 octobre 1946. 
et Seine-et-Oise le jugement de Nuremberg constituait même «un véritable déni de justice ${ }^{(87)}$ et le Comité d'action et de propagande pour le châtiment des criminels de guerre résuma: "Justice insuffisante, justice hésitante, justice confuse " ${ }^{(88)}$. Face à cette forte pression politique, le gouvernement décida finalement de réagir: le 3 octobre 1946, le garde des Sceaux Pierre-Henri Teitgen donna une conférence de presse dans laquelle il commenta le jugement du TMI et répondit aux questions des journalistes ${ }^{(89)}$. En soulignant: «Les représentants du gouvernement français ont fait non seulement tout leur devoir, mais plus que leur devoir» ${ }^{(90)}$, il chercha visiblement à dégager la délégation française de toute responsabilité par rapport aux décisions prises par le TMI. Dans ce sens L'Ordre avisa ses lecteurs le lendemain: «La France n'est pas responsable des acquittements de Nuremberg ${ }^{(91)}$. C'était bien éloigné de la vérité: le vote d'Henri Donnedieu de Vabres avait été crucial pour deux des trois acquittements et dans plusieurs autres cas le juge français avait sauvé la vie des accusés en se prononçant pour une peine de prison au lieu de la peine capitale. Mais les délibérations des juges du TMI s'étaient tenues en secret et pour cette raison on ne savait pas en France comment le jugement à Nuremberg avait été rendu. Apparemment inquiet par le mécontentement public, Donnedieu de Vabres osa même insinuer quelques jours plus tard dans une interview avec l'hebdomadaire protestant Réforme que ce n'était pas lui, mais les juges britannique et américain qui étaient responsables des acquittements ${ }^{(92)}$. Au moins, ces manouvres de dissimulation aboutirent: l'interview dans Réforme amena même Charles Dubost à croire que Donnedieu de Vabres se serait opposé aux acquittements et aux peines trop clémentes imposés par les juges anglo-américains ${ }^{(93)}$.

Ainsi, la vérité resta inconnue en France et les Français ne surent pas que leur compatriote était à l'origine de presque toutes les décisions du tribunal qui avaient causé une telle indignation générale. Jusqu’à la fin de leur vie, Donnedieu de Vabres et Falco ne se prononcèrent jamais en public sur le déroulement des délibérations et les votes des juges à Nuremberg si bien que ce secret fut bien gardé. 20 ans plus tard même, François de Menthon n'avait que des connaissances vagues et peu précises de ce qui s'était passé entre les juges du TMI. «Il arriva que pour tel ou tel accusé le juge français, le professeur Donnedieu de Vabres, joua le rôle décisif en joignant sa voix, dans le sens de la clémence, à celle du président Lord Justice Lawrence » ${ }^{(94)}$, rapporta en 1966 l'ancien procureur en chef sans pouvoir donner de détails.

87 AD, NUOI 1944-1959/91, lettre du Syndicat général du papier-carton et similaires des départements de Seine et Seine-et-Oise, 4 octobre 1946.

88 AD, NUOI 1944-1959/91, lettre du Comité d'Action et de Propagande pour le Châtiment des Criminels de Guerre, 5 novembre 1946.

89 «Le verdict de Nuremberg commenté par le garde des sceaux», Le Monde, 5 octobre 1946.

90 Pierre-Henri Teitgen, Le jugement de Nuremberg, Conférence de M. Teitgen, le 3 Octobre 1946, Paris, 1946, p. 6.

91 L'Ordre, 4 octobre 1946.

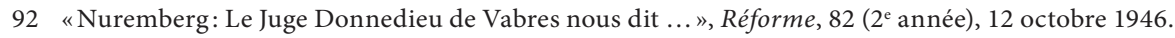

93 Charles Duвоsт, «Les crimes des États et la coutume pénale internationale», Politique étrangère, 11/6 (1946), p. 553-568, ici p. 565.

94 ADHS, 135 J 191, de Menthon, manuscrit, vers 1966, p. 7. 
À l'indignation publique s'ajouta, à long terme, la déception des Français qui avaient personnellement contribué au procès de Nuremberg. Contrairement à une grande partie des médias de leur pays, les juristes ne fustigèrent pas la clémence des juges du TMI et n'exprimèrent pas leur colère en public, mais cherchèrent à évaluer la valeur juridique du jugement et réfléchirent à ses conséquences. Leurs regards étaient clairement tournés vers l'avenir: Henri Donnedieu de Vabres souligna la "portée incomparable» ${ }^{(95)}$ du jugement pour le développement du droit pénal international. Charles Dubost vit le droit pénal international comme un "édifice qui s'élève » ${ }^{(96)}$ et auquel les événements de Nuremberg auraient posé la première pierre. Aux autres membres du parquet français, il parla de «notre combat pour la paix» ${ }^{(97)}$ qui aurait eu lieu à Nuremberg. Voués à la cause de la justice internationale, presque tous les juristes français ayant participé au procès mirent de grands espoirs dans le jugement du TMI et se montrèrent d'abord confiants dans l'idée que, suivant le modèle de Nuremberg, une juridiction internationale permanente serait instaurée par les Nations unies et serait chargée de sanctionner des crimes relevant du droit international partout dans le monde. Plein d'enthousiasme, Edgar Faure écrivit en automne 1946:

«Si les hommes d'État qui ont créé la justice internationale n'hésitent pas devant l'envergure de leur œuvre et savent y discerner la grande chance de la paix, 1946 aura mérité de demeurer dans l'histoire une date aussi mémorable que $1789{ }^{(98)}$.

Mais les juristes durent vite constater que, face aux réalités politiques, leur optimisme initial s'avéra injustifié et que la Guerre froide empêchait tout avancement du droit pénal international. «Les deux blocs qui divisent le monde semblent vouloir régler leurs rapports en force et non en droit» ${ }^{(99)}$, déplora par exemple Henri Monneray. Par la suite, les évaluations du procès de Nuremberg furent au cours du temps de plus en plus marquées par la déception des espoirs des juristes: pour Monneray les idées de Nuremberg paraissaient dès la fin des années quarante «avoir disparu sans lendemain ${ }^{(100)}$. Lors d'un colloque en 1967 Delphin Debenest fit le même constat tout en exprimant sa propre «amertume» ${ }^{(101)}$. Guidé par les mêmes sentiments, JacquesBernard Herzog donna à son livre inachevé sur le procès devant le TMI, qui fut publié après sa mort en 1968, le titre Nuremberg: un échec fructueux ${ }^{(102)}$. Certes, les juristes

95 Henri Donnedieu de VABres, «Le procès de Nuremberg devant les principes modernes du droit pénal international», in: Académie de droit international de La Haye (éd.), Recueil des cours, 70/1 (1947), Paris, 1948, p. 477-582, ici p. 577.

96 C. Dubost, «Les crimes des États» (note 93), p. 568.

97 Fondation nationale des sciences politiques (FNSP), Archives d'histoire contemporaine, CD/03, manuscrit sans titre («Je ne crois pas que la notion de crime contre l'humanité [...]»), sans date, 3 pages.

98 AN, 505 AP II/56, Edgar Faure, La justice internationale et les droits de l'homme, sans date (automne 1946), 7 pages, ici p. 7.

99 Cf. CDJC, MDXXXV/22, Henri Monneray. La justice internationale, sans date (vers 1949), 7 pages, ici p. 4.

100 Ibid., p. 3.

101 Fonds Debenest (en possession privée), manuscrit sans titre («Déjà plus d’un quart de siècle [...]»), 29 avril 1967, 11 pages, ici p. 1.

102 Jacques-Bernard Herzog, Nuremberg - un échec fructueux?, Paris, Librairie générale de droit et de jurisprudence, 1975. 
défendirent toujours la cause pour laquelle ils s'étaient engagés contre ses détracteurs: Charles Gerthoffer qualifia aussi 20 ans plus tard le procès de Nuremberg «d'œuvre grandiose" ${ }^{(103)}$ et Henri Monneray continua à considérer comme un "grand privilège » ${ }^{(104)}$ d'avoir eu la possibilité d'y participer. Mais les évaluations d'ensemble de la part des juristes restèrent dominées par la déception et le pessimisme. Tout ce qui restait du procès de Nuremberg par exemple pour François de Menthon en 1976 était «une lueur d'espoir» ${ }^{(105)}$.

Pour comprendre ce pessimisme, il faut se rendre compte que les juristes français ayant participé au procès de Nuremberg en tant que juge ou procureur ne purent voir la création de la Cour pénale internationale (CPI) à La Haye. Aucun d'eux n'était plus en vie lorsqu'en 1998 le statut de la nouvelle juridiction fut signé à la fin de la conférence de Rome ${ }^{(106)}$. C'est pourquoi le rejet du procès de Nuremberg par l'opinion publique en France se poursuivit très longtemps du fait de son évaluation pessimiste par ceux qui avaient représenté le pays auprès du TMI et qui, comme personne, pouvaient marquer de leur empreinte le débat autour de l'héritage du procès. Le résultat de ce développement fut une perception très négative dans la société française si bien que le procès de Nuremberg fut considéré comme un échec.

\section{Conclusion}

Dans l'ensemble, le rôle de la France au procès de Nuremberg peut être saisi de façon claire et nette: sur le plan politique, le gouvernement français préconisa depuis le printemps 1945 la mise en œuvre d'un procès contre les dirigeants de l'Allemagne nazie et créa, après la conférence de Londres, une délégation qui représentait le pays auprès du TMI. Comme le ministère de la Justice fut responsable du choix du personnel pour le procès de Nuremberg, on recruta surtout des magistrats qui s'étaient illustrés dans la Résistance pendant la Seconde Guerre mondiale. Ce groupe, autour du procureur en chef adjoint Charles Dubost, élabora l'accusation et en profita pour démontrer leur point de vue sur les crimes nazis en défendant surtout la thèse de la culpabilité collective des Allemands.

Pourtant, l'opinion publique en France fut clairement défavorable à l'issue du procès de Nuremberg et après le verdict un bon nombre de journaux français menèrent une campagne farouche durant laquelle ils s'emportèrent contre la clémence des juges du TMI. À ces réactions marquées par la colère et le mécontentement s’ajouta la déception des anciens membres de la délégation française pour lesquels les espoirs d'un triomphe de la justice internationale et de la création d'une juridiction permanente pour le monde entier ne se concrétisèrent pas dans les décennies suivantes. Même les juristes ayant appartenu à la délégation française en 1945 parlèrent par la suite d'un «échec » ${ }^{(107)}$

103 Ibid., p. 7.

104 CDJC, MDXXXV/24, Monneray, manuscrit sans titre («J'ai eu le grand privilège [...]»), sans date (vers 1949), 13 pages, ici p. 1.

105 ADHS, 135 J 191, de Menthon, manuscrit, vers 1976, p. 18.

106 William Sch a BAs, An Introduction to the International Criminal Court, Cambridge et al., Cambridge University Press, 2011, p. 13-22.

107 J.-B. Herzog, Nuremberg (note 102). 
qu'ils voyaient dans le procès devant le TMI. L'hypothèse selon laquelle cette perception défavorable sur différents niveaux pourrait expliquer de manière exhaustive le désintérêt manifeste que la participation française au TMI connut plus tard en France, reste à vérifier dans le cadre de recherches futures. Il faut constater ici que ce désintérêt créa une image erronée et qu'en réalité la France apporta sur le plan politique ainsi que juridique une contribution significative au procès de Nuremberg. Cette contribution mérite d'être rappelée.

\section{Résumé}

L'article analyse le rôle de la France au procès de Nuremberg en 1945/46 sous plusieurs angles: d'abord, il montre dans quelle mesure le gouvernement français suivit au printemps 1945 les préparatifs du procès et quelles positions il adopta lors des négociations avec les autres puissances alliées. Il examine ensuite la composition de la délégation française auprès du Tribunal militaire international (TMI) de Nuremberg ainsi que les interventions des juristes français pendant les audiences du tribunal. En fin de compte, l'article s'interroge sur la perception du procès de Nuremberg en France et met en évidence que celui-ci fut largement considéré comme un échec politique et juridique par la population française.

\section{Zusammenfassung}

Der Beitrag analysiert die Rolle Frankreichs im Nürnberger Prozess gegen die Hauptkriegsverbrecher aus unterschiedlichen Blickwinkeln: Zunächst zeigt er, inwieweit die französische Regierung im Frühjahr 1945 die Prozessvorbereitungen mitverfolgte und wie sie sich hierbei gegenüber den anderen alliierten Mächten positionierte. In einem zweiten Schritt werden die Zusammensetzung der französischen Delegation beim Internationalen Militärtribunal (IMT) und die Beiträge der französischen Juristen in den Sitzungen des IMT untersucht. Am Ende steht die Frage nach der Haltung der französischen Öffentlichkeit zu den Vorgängen in Nürnberg und der Aufsatz zeigt, dass der Prozess in Frankreich weitgehend als politischer wie juristischer Misserfolg angesehen wurde.

\footnotetext{
Abstract

The article examines from different perspectives the role of France in the Nuremberg Trial of the German Major War Criminals before the International Military Tribunal (IMT) in 1945/1946. It highlights first to which extend the French government followed in spring 1945 the preparation of the trial and which positions it adopted in the negotiations with the other Allied powers. Thereupon, the composition of the French delegation in Nuremberg and their contributions during the proceedings of the IMT are analyzed. In its last part the article focuses on the attitude of the French population towards the events in Nuremberg and points out that the trial was widely seen by the French as a political and judicial failure.
} 